\title{
La sustitución entre las monedas de El Salvador y Guatemala
}

\author{
Luis René Cáceres* \\ y José Roberto Suay**
}

\section{Introducción}

En años reciente, el fenómeno de la sustitución entre monedas ha merecido considerable análisis y estudio en las ciencias económicas, como resultado de las significativas implicaciones que ejerce en la conducción de la política económica. El hecho es que la teoría monetaria tradicionalmente ha presupuesto que en la economia bajo análisis circula solamente el signo monetario nacional y que, lógicamente. los individuos y las empresas demandan para fines de transacciones o de precaución, solamente esa moneda. Al introducir el supuesto de que los agentes económicos demandan monedas diferentes a la doméstica, lo que es plausible ante consideraciones de diversificación de cartera por percepciones de riesgo, o para atender transacciones especíticas, los postulados aceptados de la teoría monetaria cambian radicalmente'.

En efecto, si el individuo mantiene en cartera monedas diferentes a la nacional, cualquier factor relacionado con el riesgo o rendimiento de sus activos externos, provocaría un reajuste en su cartera, afectando la

- Funcionario del Banco Centroamericano de Integración Económica, Tegucigalpa, Centroamérica.

- Candidato al Doctorado en Econom[a, Universidad de Utah, Salt Lake, Utah, U.S.A.

Los autores agradecen los comentarios de Carlos G. Herrera, Fréderick J. Jiménez y Salvador Quintanilla a una versión preliminar de este trabajo. 
tenencia de moneda local y afeclando por ende la demanda de dinero. En consecuencia, la demanda de moneda local estaria influenciada por factores exógenos al pais en cuestión, lo cual daria lugar a una inestabilidad en dicha demanda, con serias repercusiones en la validez de paradigmas establecidos. Asimismo, dado que las monedas extranjeras pueden producir servicios como los del signo monetario doméstico, dependiendo del grado de sustitución, distintas combinaciones de monedas extranjeras y doméstica ocasionarian distintos impactos en el ingreso, de forma tal que la relación única entre ingreso y dinero (nacional) se pierde. Lo anterior provoca importantes reflexiones sobre la definición del agregado monetario más apropiado en una economia y sobre la efectividad de la programación monetaria.

La sustitución de monedas tiene también importantes consecuencias en la transmisión de impactos económicos de un pais a otros. En el caso de un régimen de tasa de cambio fija, el banco central garantiza la convertibilidad de la moneda extranjera a moneda nacional a un determinado precio (la tasa de cambio) de manera que, por el lado de la oferta, la moneda extranjera y la nacional son perfectos sustitutos. Ya que el banco central no puede controlar el monto de moneda extranjera en circulación, éste no tiene control sobre la oferta monetaria del pais, la cual es suceptible a las políticas de los bancos centrales extranjeros que afectan la disponibilidad de sus monedas en el pais en cuestión, razón por la cual las políticas monetarias de los bancos centrales son interdependientes.

En el caso de un régimen de tasa de cambio flexible, el banco central no garantiza un precio fijo de la moneda extranjera en términos de la nacional, de manera que la sustitución por el lado de la oferta no existe. Sin embargo, al introducir el supuesto de que individuos o empresas mantienen carteras diversificadas de monedas, emerge una sustitución por el lado de la demanda. Bajo esta situación, un incremento en la oferta monetaria de un pais se destina en parte a los portafolios de individuos en otros países que son usuarios de esa moneda. De esa manera, el pais estaria "desbordando" su politica monetaria expansiva, compartiendo asi sus presiones inflacionarias con las de otros paises usuarios de su moneda. Este fenómeno introduce una interdependencia en las políticas monelarias, lo cual niega validez a la hipótesis de que si un estado mantiene un régimen cambiario flexible, puede efectivamente aislar su politica monetaria de las repercusiones provenientes de otras naciones.

Entre los modelos que se han presentado para analizar este fenómeno sobresalen aquéllos orientados a detectar la magnitud de la 
elasticidad de sustitución entre un par de monedas ${ }^{2}$; otros trabajos han sido enfocados para constatar si en determinados paises existe la sustitución monetaria ${ }^{3}$. El trabajo que más controversión ha despertado es el estudio de Makinnon que argumenta que el "monetarismo doméstico" no tiene valor analítico para explicar la tasa de inflación en una muestra de países desarrollados, para los cuales la evolución de sus precios es explicada con mayor exactitud por el crecimiento de la oferta monetaria mundial, lo que se deriva del hecho de que las monedas de estos paises son sustitutos, dando lugar, por lo tanto, a que sus funciones de demanda de dinero sean inestables ${ }^{4}$. Ante este interés justificado que se ha despertado en distintos paises por la dotación e implicaciones de la sustitución entre monedas, la pregunta que necesariamente surge es si la sustitución monetaria también ocurre en economía extremadamente abiertas como las centroamericanas.

\section{EL CASO DE EL SALVADOR Y GUATEMALA}

La economia de Guatemala y El Salvador tienen varias similitudes, sobresaliendo su orientación agroexportadora, destacándose las exportaciones de café, las cuales representan más de la mitad de las ventas externas totales. Ambos paises han logrado avances en la industrialización sustitutiva de importaciones, a lo que contribuyó sin lugar a dudas la formación del Mercado Común Centroamericano (MCCA) en 1960. Su comercio reciproco ha sido considerable, alcanzando las exportaciones de El Salvador a Guatemala en los últimos anos un $60 \%$ de sus exportaciones al MCCA y un $12 \%$ de sus exportaciones totales. Las cifras correspondientes a Guatemala son de $55 \%$ y $15 \%$ respectivamente. Estudios sobre la integración económica centroamericana han apuntado que estos dos paises tienen el más fuerte vínculo comercial en la subregión centroamericana ${ }^{5}$.

En términos de integración física su vinculación es muy significativa; se puede señalar que desde 1986 está funcionando una red de interconexión eléctrica entre ambos paises; en cuanto a la red vial, existen cuatro carreteras pavimentadas que cruzan su frontera y viajar por automóvil entre las ciudades capitales sólo toma un promedio de cuatro horas. San Salvador ha sido el lugar turístico de vacaciones para los guatemaltecos, asi como para los turistas salvadoreńos lo ha sido la ciudad de Guatemala y otras numerosas ciudades de ese país. Asimismo, en los últimos años la inversión de empresarios salvadorefios en Guatemala ha sido considerable, como también lo es el "comercio informal" en ambas vias.

A partir de 1982 El Salvador inició un régimen cambiario múltiple que incluía, además del tipo de cambio oficial, una tasa del mercado banca- 
rio y una tasa de mercado (libre). El colón salvadoreño fue experimentando progresivas devaluaciones en los mrecados interbancario y libre hasta que en enero de 1986 las autoridades monetarias decretaron una devaluación oficial, estableciendo la tasa de cambio de 5 colones por dólar. El mercado libre, en cambio, continúa funcionando, pero las oscilaciones han sido menos pronunciadas que antes de la devaluación.

Guatemala también implementó un régimen de tasas de cambio múltiples, (oficial, bancario y de mercado) en 1984, depreciándose considerablemente el quetzal hasta que se estableció una nueva paridad bancaria de 2.50 quetzales por dólar en 1986.

Se debe señalar que también ha existido un mercado para el cambio de colones a quetzales y viceversa, y la difusión del quetzal en El Salvador, asi como el colón en Guatemala, es muy amplia, especialmente en tiempos de vacaciones. El Banco Central de Reserva de El Salvador ha llevado registros de las cotizaciones del quetzal en términos: de colones en la frontera de ambos paises desde enero de 1985.

Dada esa situación, es de sumo interés estudiar la sustitución eñtre los signos monetarios de El Salvador y Guatemala. La fuerte integración de sus economias, asi como su considerable integración física, hace esperar que exista sustitución entre dichas monedas y que por lo tanto existe una fuerte interdependencia en las politicas monetarias de ambos paises. Sin embargo, no se cuenta con información sobre la tenencia de colones en poder del público de Guatemala, ni sobre las existencias'de quetzales en El Salvador, por lo que no es factible estimar la elasticidad de sustitución. Asi, se recurre a olro modelo que, aunque no permite cuantificar la magnitud de la elasticidad de sustitución entre estas monedas, si permite inferir si dicho fenómeno ocurre o no.

\section{EL MODELO Y LA PRUEBA EMPÍRICA}

Existe una forma indirecta de someter a prueba la tesis de la sustitución. Se supone que si existe sustitución monetaria, el incremento en la cantidad de dinero de un país afecta el equilibrio de los balances reales, tanto de residentes como de los ciudadanos de otro país, por lo que los precios relativos permanecen intaclos. Asi, si el pais $A$ aumenta su oferta de dinero, esto dará lugar a un exceso de oferta monetaria que provoca un incremento en el nivel de los precios en $A$ y una depreciación de su moneda. Sin embargo, si la moneda $A$ es sustituto en la demanda de la moneda de un pais $B$, dicho ajuste en los precios puede ser aminorado por el flujo de la moneda de A hacia el pais $B$, lo que provoca un aumento en el nivel de precios de ambos paises en la misma proporción, por lo que la tasa de cambio permanece constante ${ }^{6}$. 
Es asi como en un mundo en que las monedas son perfectos sustitutos, las variaciones en la tasa de cambio entre dos monedas no estarian relacionados con cambios en los precios relativos. Por lo tanto, una manera de someter a prueba la tesis de la sustitución consiste en analizar si los movimientos en la tasa de cambio pueden ser explicados por variaciones en los precios de los dos paises: si la variación en la tasa de cambio no es explicada por los movimientos de los precios relativos, se puede inferir que hay sustitución.

Específicamente el modelo parte de la teoría de la paridad del poder de compra, según la cual la tasa de cambio (TC) entre las monedas de dos paises es igual a la razón de sus precios internos $(P)$; usando " $e$ " para El Salvador y "g" para Guatemala se puede expresar:

$$
\mathrm{TCe}-\mathrm{g}=\mathrm{Pe} / \mathrm{Pg}
$$

Por otra parte, recurriendo a la teoria cuantitativa del dinero, se puede representar el nivel de precios de un pais " $i$ " como:

$$
P_{1}=M_{1} V_{1} / Y_{1}
$$

En donde $P$ es el nivel de precios, $M$ la masa monetaria, $V$ la velocidad del dinero e $Y$ el ingreso nacional. Usando esta representación de los precios para ambos paises y sustituyendo en la ecuación (1) se llega a la siguiente expresión para la tasa de cambio:

$$
\text { TCe-g }=M_{\theta} V_{\theta} Y_{\theta} / M_{\theta} V_{\theta} Y_{\theta}(3)
$$

Tomando logaritmos se obtiene:

$$
\log T C e-g=\log M_{\theta}+\log V_{\theta}+\log Y_{\theta}-\log M_{g}-\log V_{\theta}-\log Y_{\theta}
$$

Finalmente, si se supone que la velocidad ingreso del dinero permanece constante, se obliene una primera versión del modelo:

$$
\log T C e-g=C+\log M_{e}-\log Y_{\theta}-\log M_{g}+\log Y_{\theta}
$$

$$
\text { en donde } C=\log V_{e} \cdot \log V_{\theta}
$$

En el caso de no haber sustitución entre el quetzal y el colón la tasa de cambio estaria perfectamente correlacionada con las variables explicativas de la ecuación (5). De tal manera, si se efectúa una regresión entre dichas variables se esperaría que los coeficientes de Me, Ye, Mg e 
Yg fueran iguales a $1,-1,-1$ y 1 respectivamente $y$, en adición, el coeficiente de determinación tendria un valor cercano a la unidad.

Una segunda versión del modelo puede derivarse de la ecuación número (4) tomando el diferencial total. En este caso se obtiene que:

$$
\frac{d T C e-g}{T C-g}=\frac{d V e}{V_{\theta}}-\frac{d V g}{V_{\theta}}+\frac{d M_{e}}{M_{\theta}}-\frac{d Y e}{Y_{\theta}}-\frac{d M g}{M_{\theta}}+\frac{d Y g}{Y_{\theta}}
$$

y tomando su aproximación en forma discreta:

$$
\% \text { TCe }-g=\% V_{b}-\% V_{\theta}+\% M_{b}-\% Y_{b}-\% M_{\theta}+\% Y_{\theta}
$$

En donde \% significa el cambio porcentual en la correspondiente variable. Si se supone nuevamente que la velocidad del dinero permanece constante, la expresión anterior puede simplificarse para obtener:

$$
\% \text { TCe-g }=\% M_{\theta}-\% Y_{\theta}-\% M_{g}+\% Y_{g}
$$

En este caso, la ausencia de sustitución también estaria relacionada con la presencia de coeficientes cercanos a la unidad y significativos en todas las variables explicativas de la ecuación y el coeficiente de determinación seria muy alto. Al contrario, entre menor sea el poder predictivo de la ecuación, la presencia de sustitución será más probable.

Ahora bien, en el caso de El Salvador y Guatemala no existen series de datos mensuales para el ingreso nacional, por lo cual se ha utilizado la cantidad de energía eléctrica generada mensualmente como variable "proxy" del ingreso nacional?. La cantidad de dinero en ambos paises fue tomada de Estadisticas FInancleras del Fondo Monetario Internacional. La cotización del quetzal en el mercado libre fronterizo de $\mathrm{El}$ Salvador, según registros del Banco Central de Reserva de ese país, tue utilizado como representativa de la tasa de cambio entre colón y quetzal. La serie mensual utilizada comprende el periodo entre enero de 1985 y diciembre de 1986.

Primero se estimó el modelo en forma logarítmica usando cuadrados mínimos ordinarios (CMO) (Ver Cuadro $N^{2} 1$ ). En este caso, el RCuadrado no es significtivo, lo que implicaria la existencia de sustitución entre el quetzal y el colón.

Se debe apuntar, sin embargo, que los resultados pueden no ser confiables debido a que en tal regresión el problema de autocorrelación 


\section{CUADRO № 1}

Versión Logaritmica del Modelo

\begin{tabular}{cccc}
\hline & CMO & AR(1) & MA(1) \\
\hline $\mathrm{C}$ & 2.70 & 1.34 & 2.99 \\
& $(1.38)$ & $(0.65)$ & $(1.38)$ \\
$\mathrm{M}_{\theta}$ & -0.25 & -0.16 & -0.23 \\
& $(-.76)$ & $(-.47)$ & $(-.69)$ \\
$\mathrm{Y}_{\theta}$ & -0.18 & -0.002 & -0.16 \\
& $(-.54)$ & $(0.01)$ & $(-.44)$ \\
$\mathrm{M}_{\theta}$ & 0.51 & 0.36 & 0.51 \\
$\mathrm{Y}_{\theta}$ & $(1.95)$ & $(1.23)$ & $(1.89)$ \\
$\mathrm{R}^{2}$ & -0.57 & -0.40 & -0.67 \\
$\mathrm{R}^{2}$ Ajust. & $(-1.55)$ & $(-1.23)$ & $(-1.67)$ \\
$\mathrm{F}^{*}$ & 0.27 & 0.37 & 0.30 \\
$\mathrm{~F}^{* *}$ & 0.08 & 0.13 & 0.05 \\
$\mathrm{DWW}^{*}$ & 1.47 & 1.56 & 1.21 \\
\hline
\end{tabular}

Nota: Números en paréntesis son los valores t.

$F^{*}$ para la regresión.

F** para la prueba de que los coeficientes son los que se esperan en ausencia de sustitución.

está presente, lo que se aprecia en un estadístico Durbin Watson de 1.38. Es así como el modelo se reestimó tratando de corregir por autocorrelación, para lo cual dos tipos de procesos autoregresivos fueron asumidos: proceso autoregresivo de primer orden AR (1) y promedio móvil de primer orden MA (1) ${ }^{\mathrm{B}}$.

En el caso AR (1) la corrección mejoró el coeficiente Durbin-Watson hasta un valor de 2.13 se incrementó el R-Cuadrado y R-Cuadrado 
Ajustado hasta 0.36 y 0.13 respectivamente; sin embargo, éstos no son estadísticamente significativos. Prácticamente la misma situación resulta cuando se asume un MA(1): el Durbin-Watson alcanzć 1.95 y el RCuadrado llega a 0.30; sin embargo, el R-Cuadrado ajustado se redujo a 0.05 y además resultó no significativo.

Para dar mayor sustentación a la tesis de la sustitución, se realizó una prueba estadistica de hipótesis conjunta de que los coeficientes de las variables explicativas en la regresión no son estadisticamente diferentes de lo esperado en ausencia de sustitución $n^{9}$. En este caso, se obtuvo un resultado favorable para la tesis de la sustitución: los valores de $1,-1,-1$ y 1 para los coeficientes de las variables $M_{0}, Y_{0}, M_{\theta}$ e $Y_{g}$ respectivamente, son estadísticamente incompatibles con los datos.

Los resultados indican que, una vez se toma en cuenta la existencia de autocorrelación, la hipótesis de que no existe sustitución entre el quetzal y el colón puede ser descartada. Aún más, la sustitución parece ser considerable: según las regresiones, ninguno de los coeficientes es individualmente significativo, el R-Cuadrado Ajustado de la regresión es bajo -alrededor del 10\%- y sobre todo en ninguno de los casos es estadisticamente significativo: el estadístico $\mathrm{F}$ no es mayor de 1.6.

A diferencia de la formulación logarítmica, en el caso del modelo en forma de tasas de crecimiento la presencia de un coeficiente Durbin Watson de 1.93 hizo innecesaria la estimación del modelo suponiendo procesos autoregresivos. (Cuadro $N^{2} 2$ ). Asimismo, los resultados de la especificación alternativa corroboran las conclusiones anteriores. EI RCuadrado resultó ser bajo $(0.14)$ y estadísticamente no significativo. Asimismo, los coeficientes de las variables tomados individualmente no son significativos. No obstante, dado que esta regresión no posee un intercepto, el uso del R-Cuadrado como criterio para determinar la bondad del ajuste es cuestionable. Es asi como se calculó el RCuadrado Momento Cero (Raw Momento R-Square), resultando ser de un valor de 0.13 , lo cual también es consistente con la tesis de sustitución.

Se efectuó nuevamente la prueba para determinar si los coeficientes de la regresión son compatibles con una muestra proveniente de una población con parámetros iguales a los que la teoría presume ante la no sustitución de monedas. Nuevamente, el estadístico $F$ fue atto (8.03), indicando que la diferencia es significativa y que por lo tanto los coeficientes son conjuntamente diferentes de $1,-1,-1$ y 1 .

L.a evidencia presentada sobre la sustitución entre el colón y el quelzal puede ser sustentada adicionalmente estirnando una ecuación 


\section{CUADRON 2}

Versionde Tasas de Crecimiento

\begin{tabular}{ll}
\hline & CMO \\
\hline$M_{\theta}$ & 0.04 \\
& $(0.11)$ \\
$Y_{\theta}$ & 0.39 \\
& $(1.19)$ \\
$M_{\theta}$ & 0.19 \\
& $(0.47)$ \\
$Y_{\theta}$ & 0.04 \\
$R^{2}$ & $(0.11)$ \\
$R^{2}$ Ajust. & 0.14 \\
$R^{2}$ Momento cero & -0.01 \\
$F^{*}$ & 0.13 \\
$F^{* *}$ & 0.90 \\
DW & 8.03 \\
\end{tabular}

Nota: Número en paréntesis son los valores t.

$\mathrm{F}^{\star} \quad$ Para la regresión.

$F^{* *} \quad$ Para la prueba de que los coeficientes son los que se esperaría en ausencia de sustitución.

para la demanda de dinero en El Salvador en función de su ingreso nacional $\mathrm{Ye}$, su tasa de inflación Pe, y el acervo monetario de Guatemala ${ }^{10}$. Una ecuación similar se estimó para Guatemala que incluia la masa monetaria de El Salvador como una variable explicativa. Los resultados son los siguientes:

El Salvador:

$$
\begin{aligned}
& \log \left(M_{20}\right)=2.8359+0.3356 \log \left(Y_{\theta}\right)+0.007 \dot{P}_{\bullet}+0.4364 \log \left(M_{2 g}\right) \\
& \text { (1.43) (1.59) (1.44) (2.01) } \\
& \mathrm{A}^{2}=0.62 \quad \text { D.W. }=2.58 \quad \mathrm{~F}=4.39
\end{aligned}
$$


Guatemala:

$$
\begin{array}{r}
\log \left(M_{2 \theta}\right)=\underset{(1.44)}{2.7402} \underset{(2.08)}{0.3205} \log \left(y_{g}\right) \underset{(2.23)}{-0.006} \dot{P}_{g}+\underset{(2.89)}{0.8177} \log \left(M_{2 \theta}\right) \\
R^{2}=0.64 \quad \text { D.W }=1.61 \quad F=4.49
\end{array}
$$

Estas ecuaciones muestran que la masa monetaria de Guatemala es la variable más significativa estadísticamente en la determinación de la demanda por $M_{2}$ en El Salvador, y similar resultado se observa en Guatemala con relación a la masa monetaria del otro pais. Estos resultados deben interpretarse con precaución, en vista del bajo valor del estadistico $F$, pero aún asi no dejan de constituir soporte a la evidencia de una sustitución entre los signos monetarios de estos dos paises.

\section{Conclusiones}

La evidencia empírica presentada en este trabajo constituye soporte a la hipótesis de que existe una sustitución entre los signos monetarios de El Salvador y Guatemala. Asimismo, el grado de sustitución puede ser muy alto, en virtud de los bajos valores del coeficiente de determinación y la ausencia de significado estadistico de las variables explicativas en las ecuaciones estimadas. Se debe tener en cuenta, sin embargo, que los resultados dependen de dos supuestos básicos: la validez de la teoría cuantitativa del dinero y de la teoria de la paridad del poder de compra ${ }^{11}$.

Las implicaciones de la existencia de una sustitución monetaria entre Guatemala y El Salvador son de gran importancia. Por una parte, se vuelve necesario a cada pais encontrar el agregado monetario que mejor explique la demanda de dinero y el proceso inflacionario. El hecho es que en EI Salvador y Guatemala no se detecta una relación directa entre la masa monetaria nacional y la tasa de inflación, por lo que es conveniente explorar en que grado un índice monetario de cada país que incluya las variables monetarias del otro, con las debidas ponderaciones y ajustes, constituiria una mejor serie monetaria "nacional". Lo anterior es de importancia especial en la programación monetaria, ya que la presencia de sustitución exigiria una programación conjunta de parte de los bancos centrales de ambos palses. En términos más generales, se podría afirmar que es necesario establecer coordinación entre las respectivas autoridades monetarias, pues, como se ha demostrado, las políticas monetarias de un país inciden en las del otro. 
1. Excelentes exposiciones sobre la sustitución de monedas se encuentran en Lance Girton y Don Roper, "Theory and Implication of Currency Substitution", Journal of Money, Credit and Banking, vol. XIII, № 1 , febrero 1981, pp. 12-30; David T. King, Bluford H. Putnam y D. Sykes Wilford, (eds.), The Monetary Approach to International Ad]ustment, New York, Praeger Publishers, 1978, pp. 199-214.

2. Véase Marc $O$. Miles, "Currency Substitution, Flexible Exchange Rates and Monetary Independence", Amerlcan Economic Review, vol. 68, № 1, junio 1978, pp. 428-436; Marc Miles y Marion B. Steward, "The Effects of Risk and Return in the Currency Composition of Money Demand", Woltwirtschaftllches Archlv, Band CXVI, 1981, pp. 613626; Jaime Márquez, "Money Demand in Open Economies" A Currency Substitution Model for Venezuela", Journal of Internatlonal Money and FInance, vol. 6, 1987, pp. 167-178.

3. Véase Bruce Brittain, "International Currency Substitution and the Apparent Instability of the Velocity is some Western European Economies and in the United States", Journal of Money, Credit and BankIng, vol. XIII, N2 2, mayo 1981, pp. 135-155; Michael Bordo y Eshan U. Choudhri, "Currency Substitution and the Demand for Money: Some Evidence for Canadan. Journal of Money Credlt and Banklng, vol. XIV, NN 1, tebrero 1982, pp. 48-57; Guillermo Ortiz, "Currency Substitution in México: The Dollarization Problem", Journal of Money, Credit and Banking, vol. XI. № 2, mayo 1983, pp. 174-185.

4. Ronald I Makinnon, "Currency Substitution and Instability of the World Standard", Amerlcan Economlc Revlew, vol. 72, junio 1982, pp. $320-$ 330.

5. Luis René Cáceres y Carlos Arturo Imendia, "Vinculación Comercial e Integración Económica en Centroamérica: Una Aplicación del Análisis de Flujo de Transacciones",. Cuadernos de Economla y Flnanzas, № 2. Banco Centroamericano de Integración Económica, 1987.

6. Este modelo es expuesto on Arthur B. Laffer y Marc A. Miles Intenational Economics, Glenview, Scott, Foresman and Company, 1982, capitulo 19.

7. La generación mensual de energla eléctrica se ha tomado como una "proxy" del ingreso nacional mensual en base a un estudio del Banco Central de Reserva de El Salvador que demuestra que la generación de energla eléctrica es la variable más importante en la trimestralización del producto interno bruto anual. Veáse Mauricio González Orellana, "La Trimestralización del PIB de El Salvador", Banco Central de Reserva de El Salvador, 1983, mimeo.

8. En el proceso autoregresivo de primer orden se supone que el error de la regresión puede representarse como:

$$
\theta_{1}=e_{1.1}+U_{1}
$$


En cambio, en el promedio móvil de primer orden se asume que el error de la regresión es un promedio entre el error puro "pure noise" actual y el error puro del perlodo anterior:

$$
\theta_{1}=U_{1}+U_{t-1}
$$

(Ver Jan Kmenta, Elements of Econometrics, New York, MacMillan, 1971).

9. Si se llama $\mathrm{SEC}_{\mathrm{u}}$ a la suma de residuos al cuadrado que resulta de la regresión:

$\log T C_{a / g}=b_{0}+b_{1} \log M_{0}-b_{2} \log Y_{0}-b_{3} \log M_{9}+b_{4} \log Y_{0}+u$

Y SEC, a la suma de residuos al cuadrado que resulta de la misma regresión pero sujeta a las siguientes restricciones:

$$
b_{1}=1 ; b_{2}=-1 ; b_{2}=-1 ; b_{3}=1 ; b_{4}=1
$$

Entonces, la hipótesis de que los coeficientes tienen los valores anteriores (que la teoría ortodoxa es correcta) puede ser validada mediante una prueba $F$, en donde $F$ toma el valor:

$\left(S E C_{1}-S E C_{u}\right) \times(T-K) / S E C_{u} \times J$

Donde $J$ es el número de hipótesis independientes que se someten a prueba; $T$, el número de observaciones; y $\mathrm{K}$, el número de coeficientes en la regresión. $J$ y T.K, además, representan el número de grados de libertad on el numerador $y$ denominador correspondiente. Si $F$ es significativa para $\mathrm{J}$ y $\mathrm{T}-\mathrm{K}$ grados de libertad la hipótesis anteriores es rechazada.

10. En las ecuaciones se utilizan datos trimestrales de los años 1984, 1985 y 1986. La generación de energla eléctrica se usa como "proxy" del ingreso en ambos palses.

11. El uso de tasas de cambio en el mercado libre en el contexto de la partida del poder de compra se puede justificar a la luz del estudio de William Patton Culberton, "Purchasing Power Parity and Black Market Exchange Rate", Economic Inquiry, vol. XIII, № 2, junio 1975, pp. 287296. 